\title{
Date of Reference Time Point
}

National Cancer Institute

\section{Source}

National Cancer Institute. Date of Reference Time Point. NCI Thesaurus. Code C158111.

The date for a fixed reference time point. 